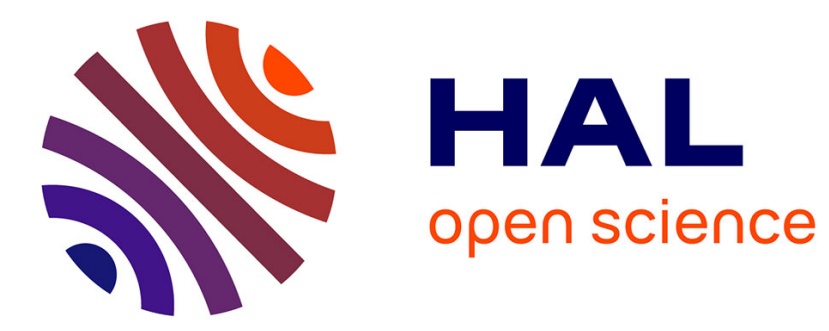

\title{
Atomic structure, strain and chemical composition at the nanometric scale in Ge/Si quantum effect devices
}

Dalil Brouri, Jean-Yves Laval, Marcin Zak, Claude Delamarre

\section{To cite this version:}

Dalil Brouri, Jean-Yves Laval, Marcin Zak, Claude Delamarre. Atomic structure, strain and chemical composition at the nanometric scale in Ge/Si quantum effect devices. Philosophical Magazine, 2006, 86 (15), pp.2151-2161. 10.1080/14786430600640478 . hal-00513673

\section{HAL Id: hal-00513673 \\ https://hal.science/hal-00513673}

Submitted on 1 Sep 2010

HAL is a multi-disciplinary open access archive for the deposit and dissemination of scientific research documents, whether they are published or not. The documents may come from teaching and research institutions in France or abroad, or from public or private research centers.
L'archive ouverte pluridisciplinaire $\mathbf{H A L}$, est destinée au dépôt et à la diffusion de documents scientifiques de niveau recherche, publiés ou non, émanant des établissements d'enseignement et de recherche français ou étrangers, des laboratoires publics ou privés. 


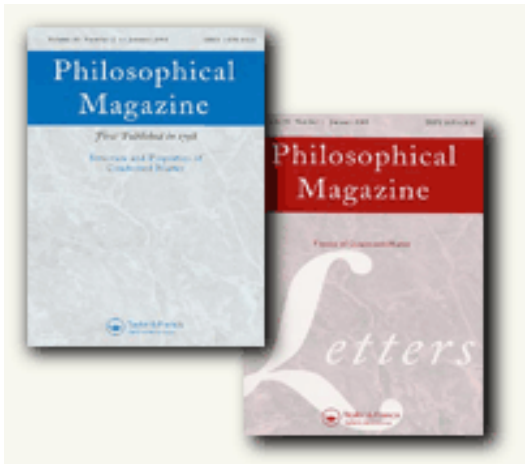

\section{Atomic structure, strain and chemical composition at the nanometric scale in Ge/Si quantum effect devices}

\begin{tabular}{|r|l|}
\hline Journal: & Philosophical Magazine \& Philosophical Magazine Letters \\
\hline Manuscript ID: & TPHM-05-Apr-0124.R1 \\
\hline Journal Selection: & Philosophical Magazine \\
\hline $\begin{array}{r}\text { Date Submitted by the } \\
\text { Author: }\end{array}$ & 06-Feb-2006 \\
\hline Komplete List of Authors: & $\begin{array}{l}\text { Brouri, Dalil } \\
\text { Laval, Jean-Yves; LPS ESPCI } \\
\text { Zak, Marcin } \\
\text { Delamarre, Claude }\end{array}$ \\
\hline Keywords (user supplied): & quantum dots, deformation, HRTEM \\
\hline
\end{tabular}

\section{ScholaronE \\ Manuscript Central}




\begin{abstract}
Germanium quantum dots were grown by Ultra High Vacuum Chemical Vapour Deposition on $\{100\}$ silicon substrates. Two types of heterostructures were studied: single islands randomly grown on silicon as well as stacking islands grown in between silicon spacers. $\{110\}$ cross-sections were prepared by mechanical polishing for high resolution electron microscopy observation (HREM) and X-ray nanoanalysis with a $200 \mathrm{kV}$ transmission electron microscope equipped with a field emission gun. Two methods were considered for measuring the local displacements from the HREM images. The first consists of localizing the center of the atomic columns with sub-pixel resolution by image processing mostly in real space. In the second method, the displacements were measured through the local Fourier components of the lattice fringes, by calculating two-phase images from two reciprocal vectors. Strains, stresses and germanium concentrations were then deduced from the displacements. Only calculations leading to comparable results from both methods were retained. Quantitative X-ray nanoanalysis was carried out by refining the Cliff-Lorimer factors for $\mathrm{Ge}$ with a tri-layered template calibrated by measuring the different $\mathrm{Ge}$ concentrations via Rutherford back scattering. A comparison of these complementary data leads to a well-documented and quantitative description of the atomic structure, the local deformations and the chemical composition in the heterostructures and reveals where interdiffusion occurs.
\end{abstract}

\title{
1. INTRODUCTION.
}

The miniaturization of IV-IV devices increases tremendously the role of stressinduced effects on the applied properties [1]. Mechanical stresses act in an ambivalent way: they may often constitute a technological lock but they can also open new fields of application as it is the case with quantum structures. Several stages in the processing of these devices can deeply modify the electronic properties but, up to now, the exact part played by local mechanical stress is not known. In particular, interdiffusion and segregation of species can interfere with mechanical effects. Therefore, to understand and optimise the properties of these components, one needs to gain combined information on the atomic structure, stress field and chemistry with a lateral resolution down to the smallest dimension of the components i.e. $1 \mathrm{~nm}$. This can be carried out only by High Resolution Transmission Electron Microscopy (HRTEM) and analytical TEM with a field emission gun (FEG). We combined $\mathrm{X}$-ray nanoanalysis and high resolution observations with image processing in order to extract local deformations in silicon germanium quantum dots.

\section{EXPERIMENTAL CONDITIONS}


Germanium quantum dots were grown at the Institut d'Electronique Fondamentale, Orsay [2,3]. The growth process was carried out by Ultra High Vacuum Chemical Vapour Deposition (UHV-CVD) on $\{100\}$ silicon substrates. The pressure of the chamber was around $10^{-10}$ Torr. The precursor gases were silane and germane diluted in hydrogen. We have investigated two kinds of Si-Ge heterostructures:

- Single Ge islands randomly distributed on the substrate. Sample A1 was grown at $700{ }^{\circ} \mathrm{C}$ with four monolayers (4 ML) of Ge deposited and sample A2 was grown at $800{ }^{\circ} \mathrm{C}$ with five to six ML of Ge deposited $(1 \mathrm{ML}=0.1457 \mathrm{~nm})$.

- Stacking islands (sample B1 and B2): In sample B1, dots were grown by selective epitaxial growth (SEG) on a patterned silicon $\{100\}$ substrate at $650^{\circ} \mathrm{C}$. The motif is a succession of lines and vias and we specifically observed islands in $0.4 \mu \mathrm{m}$ wide lines along $\langle 100\rangle$. Six ML's were deposited for the first layer and four ML's from the second to the seventh leading to a stacking of seven Ge dots separated by $9 \mathrm{~nm}$ thick Si layers. In sample B2, five Ge layers were self-assembled on the entire surface with constant Ge quantities separated by Si spacer layers of $22 \mathrm{~nm}$. The islands obtained are randomly distributed on the surface and selfassembled vertically.

Thin-films with $\{110\}$ cross-sections were prepared for HREM observations and nanoanalysis in TEM by mechanical polishing (tripode technique) perpendicular to the epilayer. The observations were carried out with a JEOL 2010 FEG electron microscope operating at $200 \mathrm{kV}$ equipped with a field emission gun and a PGT X-ray selective energy analyser. HREM images were obtained along $<110>$ directions with an objective aperture corresponding to a cut-off of $29 \mathrm{~nm}^{-1}$. This aperture gives a point resolution of $0.19 \mathrm{~nm}$ and an information limit of $0.10 \mathrm{~nm}$. Focal series were carried out to obtain the optimal defocus corresponding to a large area of dotted contrast. Simulations of the HREM images were performed with the JEMS software and showed that under such conditions large uniform areas with stable white dots (intensity maxima in images) could be obtained for a wide range of foil thickness and defocus values. We obtained experimentally and by simulations an optimal contrast for a defocus close to 1.2 times the Scherzer defocus in agreement with previous results [4]. The images were digitized with a sampling of 4 pixels/ $\AA$ and 8 bit dynamics. Parts of images corresponding to $50 \mathrm{~nm}$ per $50 \mathrm{~nm}$ were processed. Image processing was achieved with routines written in Optimas v 4.0, Analytical Language for Images (ALI) and Spyglass Transform on a Pentium based computer following an algorithm which includes Wiener Fourier filtering with local noise estimation and high-low frequency removal. This procedure conserves information on lattice positions and improves signal to noise ratio. The stress field was obtained in two ways: (a) Intensity maxima localisation and (b) the "Geometric phase method", as described below:

a- In order to obtain deformations from HREM images, we need a sub-pixel resolution in the localization of intensity maxima. This is obtained by the procedure proposed by Rosenauer et al [5] with a fit of four parabolas around each pixel of maximum intensity [6]. The processed area should contain a non-deformed zone where the lattice parameters were calculated by a Newton optimisation method on 5000 atomic columns. The template and deformed lattice were built up with a common origin and the displacement field was calculated at each experimental lattice point. A continuous field was then obtained by projection on a rectangular grid via interpolation and averaging with nearest neighbours [7]. Experimental strain maps were deduced by derivation. 
b- The "geometric phase" approach combines real and reciprocal space. Displacements are measured by calculating the local Fourier components of the lattice fringes. The phase of each of the Fourier components is simply related to the displacement. By calculating two-phase images for two different reciprocal vectors the displacement field is obtained [8]. To avoid artefacts in the calculations only images giving the same strains with these two methods are taken into account.

The conditions, for which the maxima of intensities represent effectively the $\{110\}$ projection of the atomic structure and preserves exactly the interatomic distances, have been described previously [7, 9]. On the other hand, as far as the "geometric phase method" is concerned, Plamann et al [10] have shown that for centro-symmetrical structures, astigmatism and changes in thickness do not influence the values of displacements. Furthermore, experimental strain field maps obtained via the 2 methods were compared with those obtained by finite elements calculations in $\mathrm{GaAS} / \mathrm{Ga}_{1-\mathrm{x}} \mathrm{In}_{\mathrm{x}}$ As systems [11] using a continuous elasticity isotropic model. This enabled us to draw maps representing the indium composition. Effectively, these maps were in good agreement with X-ray nanoanalysis [12]. Consequently, these two methods can be applied to the determination of displacement, strain, stress and concentration in $\mathrm{Si} / \mathrm{Si}_{1-\mathrm{x}} \mathrm{Ge}_{\mathrm{x}}$ systems.

X-ray nanoanalysis was performed in EDX-TEM mode with a $1 \mathrm{~nm}$ probe. The CliffLorimer factors were refined by measuring the Ge concentration on a calibrating sample. This consists of an heterostructure containing 3 germanium layers with germanium concentration of 11, 13 and 16 at\% measured by Rutherford Back Scattering. The thicknesses of the layers were $120 \mathrm{~nm}, 120 \mathrm{~nm}, 50 \mathrm{~nm}$ respectively and $50 \mathrm{~nm}$ for the silicon spacer. The calibrating sample was prepared under the same experimental conditions as used for the $A_{1}, A_{2}, B_{1}$ and $\mathrm{B}_{2}$ samples. Excellent agreement was obtained on two of the layers and a mismatch of 1.5 at $\%$ for the third one. This could be due to border effects on the sample.

\section{GERMANIUM CONCENTRATION}

In order to analyse silicon and germanium interdiffusion in the quantum dots, the concentration of species were measured. For this purpose quantitative X-ray measurements were performed on a large number of quantum dots (about 30 for single islands and more than 10 for stacking islands). The quantum dots were probed along and normal to the growth direction at several points and the results are reported on representative islands.

\section{A. SINGLE ISLANDS:}

We observed hemispherical islands (Fig. 1) with a $100 \mathrm{~nm}$ width and a $20 \mathrm{~nm}$ height for sample A1 and $250 \mathrm{~nm}$ by $45 \mathrm{~nm}$ for sample A2. X-ray nanoanalysis measurements were performed and are reported on the TEM images (Fig. 1 and 2). Diffusion of silicon inside islands is found and there is not a very significant variation in the composition. The Ge concentration is approximately 50-55 at \% in sample A1 and 40 at \% in A2. However, in addition to hemispheric form we found, in sample A2, islands with a shape intermediate between a dome and a pyramid (Fig. 2). As we can see in the TEM image, it has a truncated pyramid base of $300 \mathrm{~nm}$, is longer than the "dome" islands, and $40 \mathrm{~nm}$ high surrounded with a $20 \mathrm{~nm}$ high dome. The composition is in the same range (a few \% less) with a maximum Ge concentration around $40 \%$.

\section{B. STACKING ISLANDS:}


Observations of cross sections along $\{110\}$ were achieved on sample B1. We observed isolated islands (Fig. 3a) and two adjacent islands (Fig. 3b). The Ge layers are perfectly ordered vertically as was observed previously by Xie et al. [13] in GaAs/InAs quantum dots and in SiGe [14, 15]. However there are anomalies in the shape and dimension for the first three layers. We can see that the spacer Si layer disappears between the three first layers whereas it is well established for the last layers $(4,5,6,7)$. The nanoanalysis of heterostructure B1 (selective epitaxial grown islands) is reported in Fig.4. There is an interdiffusion zone in the first three layers and the Ge concentration does not exceed $28 \%$. The concentration is maximum $(40 \%)$ in the $4^{\text {th }}$ layer and decreases smoothly down to $35 \%$ in the last layer. After the third layer the silicon layer is clearly distinguishable and there is no Ge interdiffusion between layers. The impacts of the nanoanalysis electron probe are visible in the different layers. In sample B2 the growth of quantum dots occurs on the whole surface of the substrate. The islands are 4-6 $\mathrm{nm}$ high with a spacer layer of $22 \mathrm{~nm}$. As the Ge content was maintained constant, we observed an increase in island length from $100 \mathrm{~nm}$ in the first layer to $130 \mathrm{~nm}$ for the fifth one. Such an increase was reported in similar compounds by Le Thanh et al [16] and is a limitation for applications. Nanoanalysis measurements are reported in Fig. 5. We present data in the middle of the islands and laterally for each layer. The Ge concentration increases smoothly from $15 \%$ in the first layer to $38 \%$ in the fifth and the lateral concentration follows the same evolution with lower values (from $9 \%$ to $26 \%$ ). It appears that although the islands are vertically stacked, there is dispersion in dimension and composition.

\section{STRAIN CALCULATIONS FROM HIGH RESOLUTION IMAGES}

HREM images of the islands were analysed to extract local deformations and composition by image processing. The procedure aimed to obtain optimal HREM images but this could be achieved only in the case of stacking islands (B1). For single islands, it was not possible to obtain very thin areas $(<20 \mathrm{~nm})$ for high resolution images because the islands are not covered as in stacking islands and are affected by polishing.

In Fig. 6 is shown an HREM image with a stacking of seven dots. Only half of the structure is represented. The two methods, localization of intensity maxima and the "geometric phase method" were used for the calculation of strains. Processed areas are represented by white squares. The strains $\varepsilon_{X}$ along $<110>$ ( $x$-axis) perpendicular to the growth direction ( $z$-axis) are negligible for each processed area as shown in the cartography (Fig. 7). Strain maps along the growth direction $\varepsilon_{Z}$ are displayed in Fig. 8. A variation of 0.030 to 0.070 is observed in $\mathrm{Ge}$ whereas the strains are negligible in the Si layers. Except in the first three layers there is no diffusion between Ge layers. Since strains are negligible along the $x$-direction, stresses are created only in this direction and totally relaxed along $z$. Hence

$$
\sigma_{x}=\sigma_{y}=\sigma \quad \text { and } \sigma_{Z}=0
$$

where $\sigma_{x}, \sigma_{y}$ and $\sigma_{Z}$ correspond to the stresses along [100], [010] and [001] respectively. Elasticity equations in the $\mathrm{Si}_{1-\mathrm{x}} \mathrm{Ge}_{\mathrm{x}}$ reference give:

$$
\varepsilon_{x}=\varepsilon_{y}=-\frac{1-v}{E} \cdot \sigma=-\cdot \frac{\Delta a}{a} \quad \varepsilon_{Z}=\frac{2 \cdot v}{E} \cdot \sigma
$$


where $\varepsilon_{x}, \varepsilon_{y}$ and $\varepsilon_{Z}$ are the corresponding strains, $a$ is the pure Si lattice parameter and $E$ and $v$ are Young's modulus and Poisson's coefficient. The difference in lattice parameter between the alloy and pure silicon is given by

$$
\Delta a=a_{S i_{1-x} G e_{x}}-a_{S i}
$$

Hence relative to the pure silicon reference these equations become:

$$
\varepsilon_{x}=\varepsilon_{y}=0 \quad \varepsilon_{Z}=\frac{1+v}{1-V} \cdot \frac{\Delta a}{a}
$$

Now $\frac{\Delta a}{a}=0.042 \cdot x$ where $x$ corresponds to the Ge concentration in $\mathrm{Si}_{1-\mathrm{x}} \mathrm{Ge}_{\mathrm{x}}$. Since Poisson's coefficient is in the interval [0.27, 0.28], the Ge concentration deduced from the strain $\varepsilon_{\mathrm{z}}{ }^{\prime}$, can be written:

$$
x \approx 13.57 \cdot \varepsilon_{z}^{\prime}
$$

From the strain maps, the Ge concentration was calculated for each layer and the result is reported in Fig. 9. The Ge concentration could be calculated only in the central part (white squares in Fig. 6) of the heterostructure which exhibits quite a regular contrast. This concentration is then found to be constant. It was shown by X-ray nanoanalysis that the Ge concentration decreases slightly towards the edges of the islands.

\section{DISCUSSION}

Diffusion of silicon inside the islands along the growth direction is found in both single uncovered and stacking islands but there is no diffusion of germanium inside the silicon spacers in stacked quantum dots. In uncovered islands, the Ge concentration was found to be nearly constant and decreases with the island's dimensions $(50-55$ at\% for $\sim 100 \mathrm{~nm}$ length to $40 \%$ for $\sim 250 \mathrm{~nm}$ length). However, in stacking islands, X-ray nanoanalysis measurements show also that there is Ge segregation inside the islands. The Ge concentration decreases by $10 \%$ from the centre to the side in each layer. In stacking islands both diffusion and inter-diffusion were found. By X-ray nanoanalysis it was shown that in both selfassembled and SEG islands, Ge concentration increases for the first layers and then decreases slightly. Inter-diffusion is present only in SEG structures where the Si layer between islands becomes a few nm thick.

The germanium concentration in SiGe stacking islands measured by $\mathrm{X}$-ray nanoanalysis was compared to calculations from HREM images. Except in the first two layers where interdiffusion occurs, the results are comparable using the two methods. There is good agreement for layers 3, 4, 7 but there is a $10 \%$ difference between the two results for the fifth and sixth layers. This mismatch can be attributed to a topography effect that we found in the calibration sample and/or artefacts in the HREM image processing. Effectively, it should be noted that the calculations were carried out on areas of $50 \mathrm{~nm}$ by $50 \mathrm{~nm}$, so it was not always possible to process simultaneously the islands and substrate. In this case, the silicon layers were taken as the template. This approximation was tested for the first layers where it was possible to carry out and compare the procedure with the substrate as well as with the $\mathrm{Si}$ layers. 
Selective epitaxial growth is assumed to give islands with uniform size and shape but, as found by electron microscopy, it is not the case for the first two layers due to interdiffusion. The growth conditions need then to be modified to optimise the structure. In this way, an interesting hypothesis was introduced by Le Than et al [16]. They attributed the increase in island length to a decrease in critical thickness induced by the stress field. Therefore Ge concentrations in the layers were adjusted to obtain "identical" islands (six ML's in the first and four in the others). However, this did not seem to be sufficient. Interdiffusion in the first germanium layers could be limited by increasing the thickness of the Si spacer layers but this could have influenced the critical thickness by stress field variations. Thus, we calculated the stress field from the strain maps. It was found to be negligible along the growth direction whereas values ranging from 2 - $7 \mathrm{GPa}$ were found perpendicular to this direction. These rather high stresses could effectively induce size variations in the stacking islands.

\section{Conclusion}

We investigated single islands and stacking islands (self-assembled and selective epitaxial grown) quantum dots. The germanium concentration was measured by X-ray nanoanalysis and calculated by image processing on $\{110\}$ HREM images. We obtained fair agreement between the two methods. We mostly observed diffusion of silicon towards the germanium layers and interdiffusion is usually small between the heterolayers. However, strong interdiffusion was found in the first layers of the stacking islands. On the single islands, which were uncovered, a quasi uniform concentration was measured whereas in the stacking islands an increase in Ge concentration was observed towards the center of the islands. Changes in the shape and dimensions of the islands were found by HREM observations and give useful information on how to improve the properties of these quantum dots.

The juxtapostion of concentration maps obtained directly by X-ray nanoanalysis in FEG-TEM and by extrapolation of HREM experimental strain maps using elastic constants appears to be very efficient. With this approach, one can investigate the experimental factors that could give rise to artefacts. This leads to more precise measurements and an improvement in the reliability of the data. Finally, the combination of the two methods gives a better understanding of the atomic phenomena occurring during the growth of $\mathrm{Si} / \mathrm{Ge}$ nanostructures which is key for optimising silicon germanium quantum devices.

\section{ACKNOWLEDGEMENTS}

We are very grateful to D. Bouchier and Lam H Nguyen (Institut d'Electronique Fondamentale-Orsay) for the UHV CVD growth of the SiGe quantum dots. 


\section{REFERENCES}

1. D.J. PAul, Semicond. Sci. Technol. 19 R75-R108 (2004)

2. P. Boucaud, V. Le Than, V. Yam, S. Sauvage, N. Meneceur, M. Elkurdi, D. DÉBARRE, D. BOUCHIER, Materials Science and Engeenering B89 36-44 (2002)

3. H. Nguyen Lam, H. Le Than Lam, H. Yam lam, D. Debarre, M. Halbwax. and D. BOUCHIER, Phys. Stat. Sol. (a) 201, Nº 2 353-356 (2004)

4. M.A. O’KeEFe, Ultramicroscopy 47 , 282-297 (1992)

5. A. Rosenauer , S. Kaiser, S. Reisenger, T. Zweck, W. Gebhard and D. Gerthsen, Optik (Stuttgart) 1011 (1996)

6. R. Bierwolf, M. Hohenstein, F. PhilipP, O. Brandt, G. Crook and K. Ploog, Ultramicroscopy 49 , p.273 (1993)

7. S. Kret, C. Delamarre, J.Y. Lava And A. Dubon, .Phil. Mag. Lett. 77 249-256 (1998)

8. M. HŸTCH AND P. BAYLE Electron Microscopy 2A, Editions de Physique, p.129-130 (1994)

9. S. Kret PhD Thesis, Université PARIS VI (1998)

10. T. Plamann, M.J. HŸtch, S. Kret, J.Y. Laval and C. Delamarre, Institute of Physics Conferences Series, 146 Institute of Physics, London, 23-26 (1999)

11. S. Kret, T. Benabbas, C. Delamarre, Y. Androussi, A. Dubon, J.Y. Laval and A. Lefebvre, J. Appl. Phys. 86, 1988-1993 (1999)

12. J.Y. Laval, S. Kret, C. Delamarre, P. Bassoul, T. Benabbas and Y. Androussi Microscopy and Microanalysis 8 , 312-318 (2002)

13. Q. XIE., A. Madhukar , P. Chen And N.P. KobAyashi, Phys. Rev. Lett., 752542 (1995)

14. J. Tersoff And F.K. LeGoues, Phys. Rev. Lett, 723570 (1994)

15. P. Schittenhelm, G. Abstreiter, A. Darhuber, G. Bauer, P. Werner And A. Kosogov, Thin. Solid. Films, 294291 (1997)

16. V. Le Than, V. Yam, Y. Zheng And D. Bouchier, Thin Solid Films 280 2-9 (2000) 

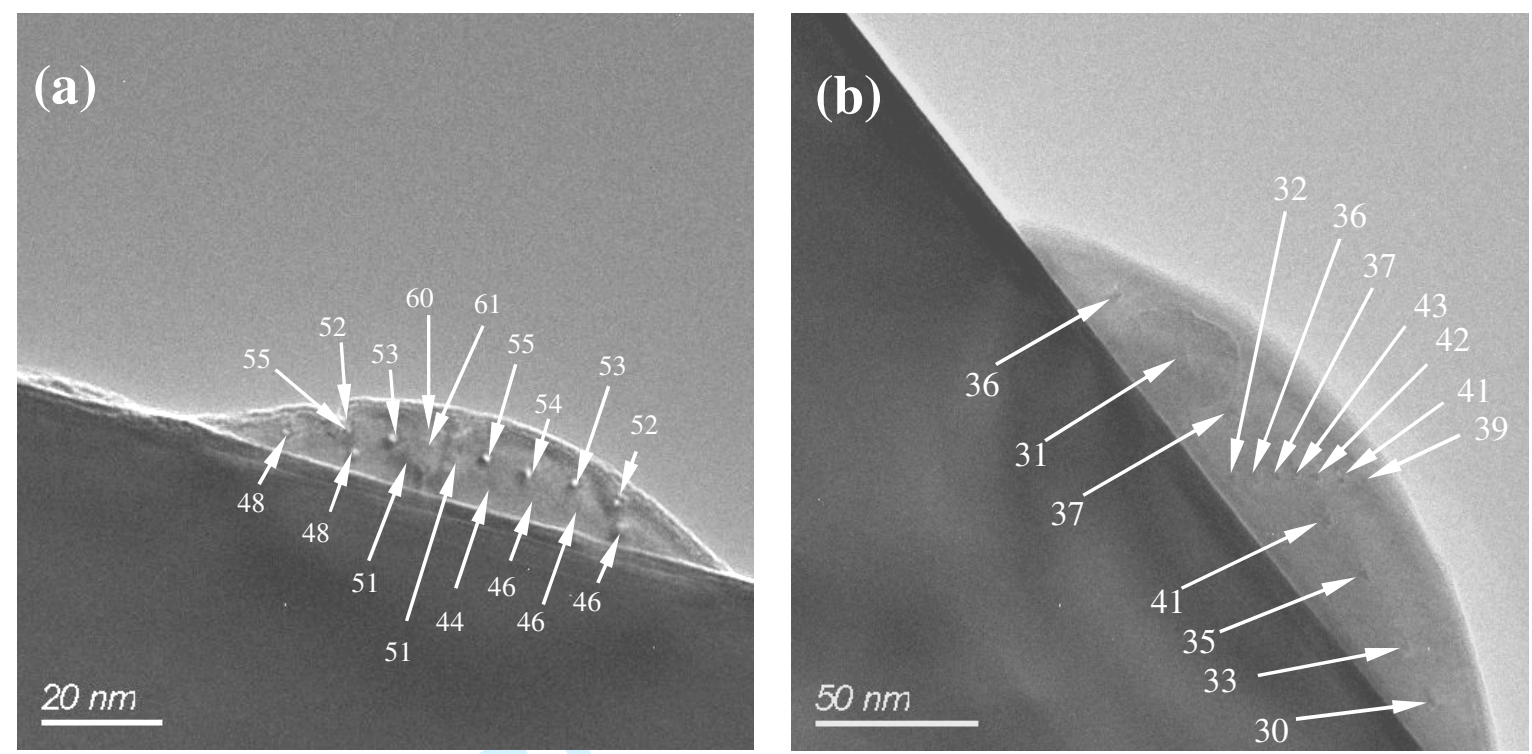

Fig. 1

TEM image and X-ray nanoanalysis on single island quantum dot A1 (a) and A2 (b). Islands have mostly a dome-like shape and the Ge concentration is found to be about $50-55$ at. $\%$ for $\mathrm{A} 1$ and 40 at $\%$ for $\mathrm{A} 2$

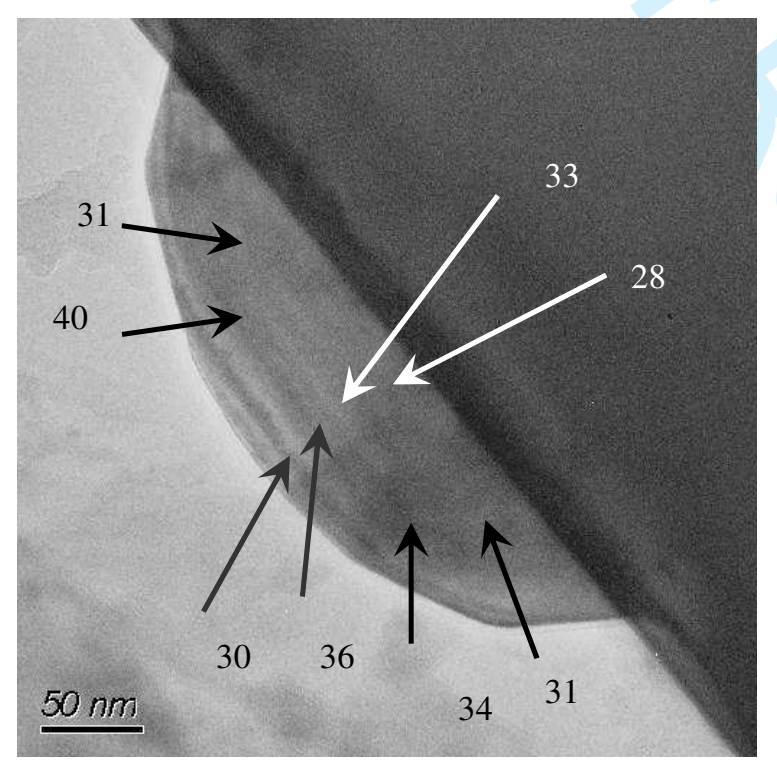

Fig. 2: TEM image and X-ray nanoanalysis measurements on single island quantum dot A2. Some islands have intermediate shape between a dome and a pyramid. The Ge concentration has the same value as in the dome-like islands 

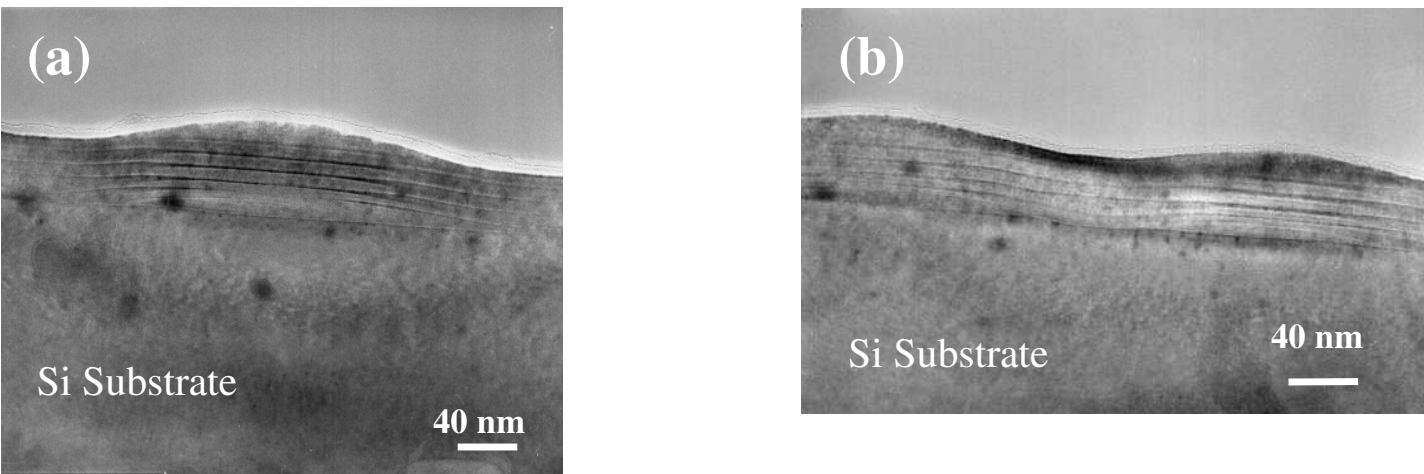

Fig. 3

Cross section TEM image of stacking islands quantum dots (B1) grown on a patterned substrate. Depending on pattern dimensions one island (a) or two adjacent islands (b) are obtained. The contrast is reversed in comparison with images below (fig. 4, 5, 6, 8, 9) obtained at higher magnification.

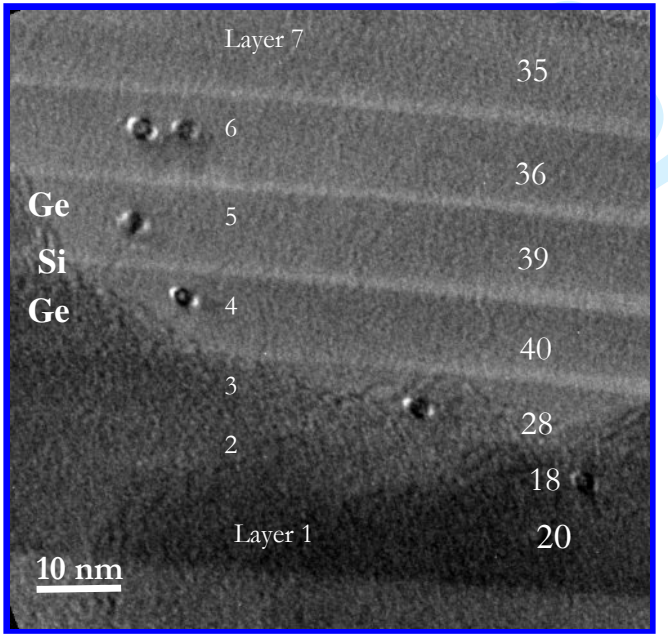

Fig. 4

X-ray nanoanalysis carried out on sample B1 for six layers. The local points in the layers are due to electron impacts and the large dark area at the bottom of the image is due to contamination as the image is obtained after analysis.

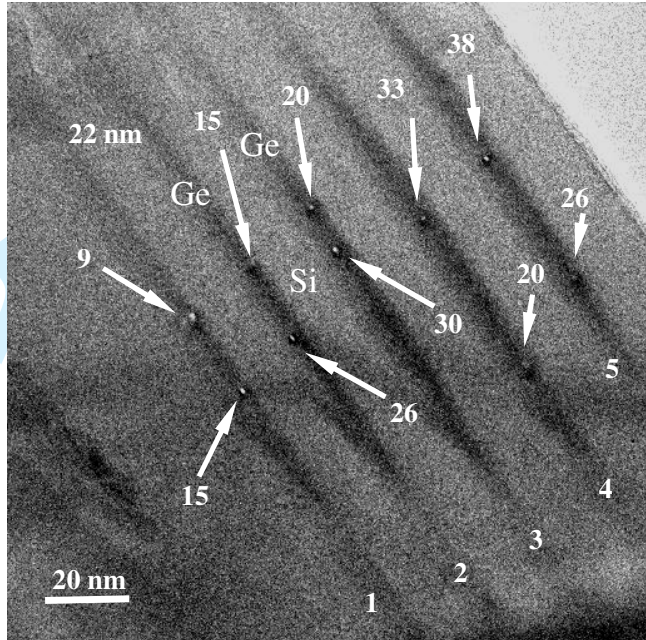

Fig. 5

$\mathrm{X}$-ray nanoanalysis measurements on stacking islands quantum dots (B2). The island length increases from the first layer to the last layer and concentration is largest in the fifth layer (38 at\%) 
Fig. 6

HRTEM image obtained on 7 island stacking (sample B1). Only the right half of the structure is shown. White squares correspond to the area analysed by image processing $(50 \mathrm{~nm}$ per $50 \mathrm{~nm})$

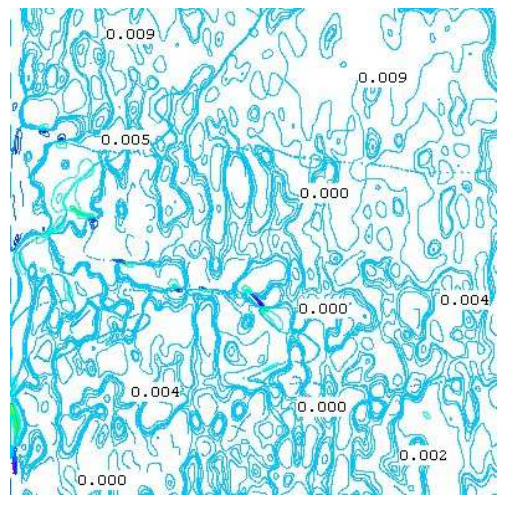

Fig. 7

Strain map: $\varepsilon_{X}$ along $<110>$, perpendicular to growth direction.
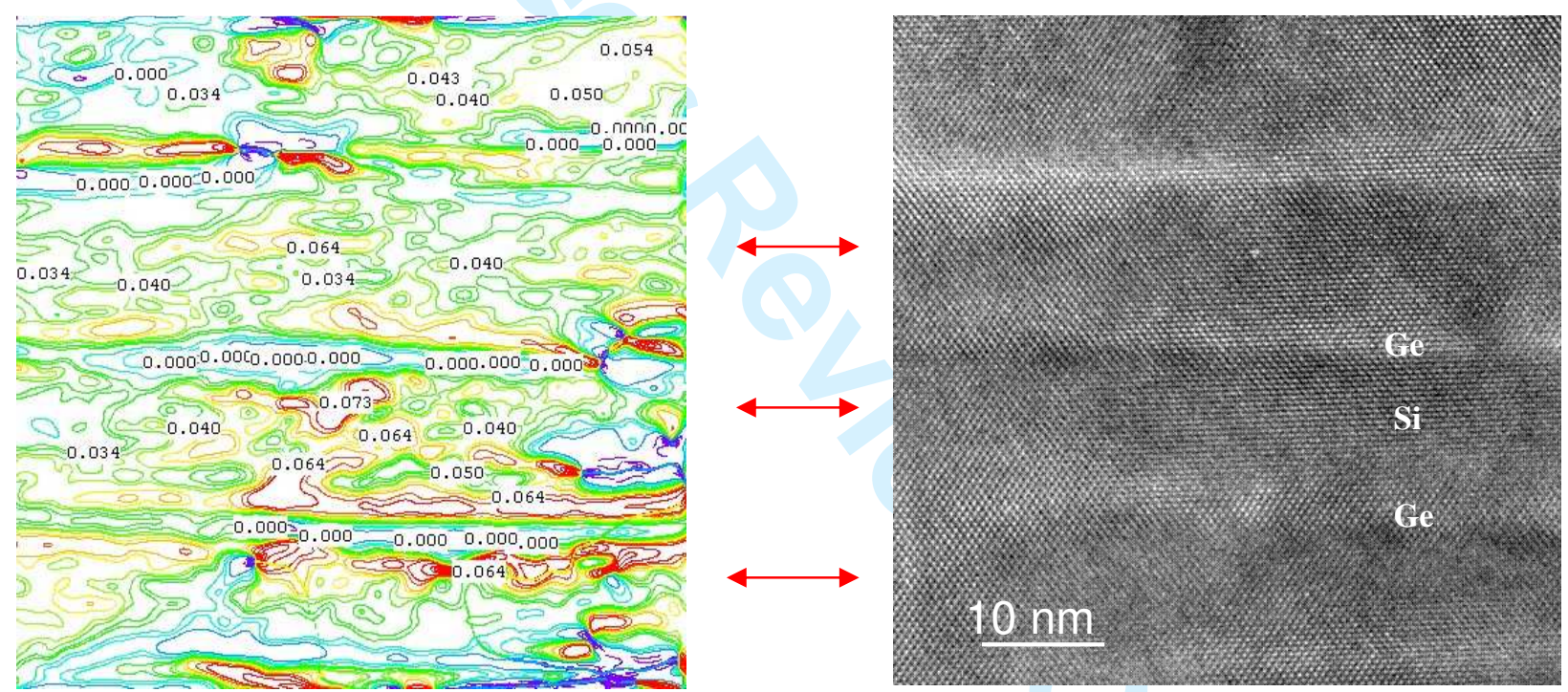

Fig. 8

Strain map $\varepsilon_{Z}$ along the growth direction and HREM image corresponding to the processed area. Strain inside germanium is in the range $0.030-0.070$ and is minimum in silicon spacer layers. 


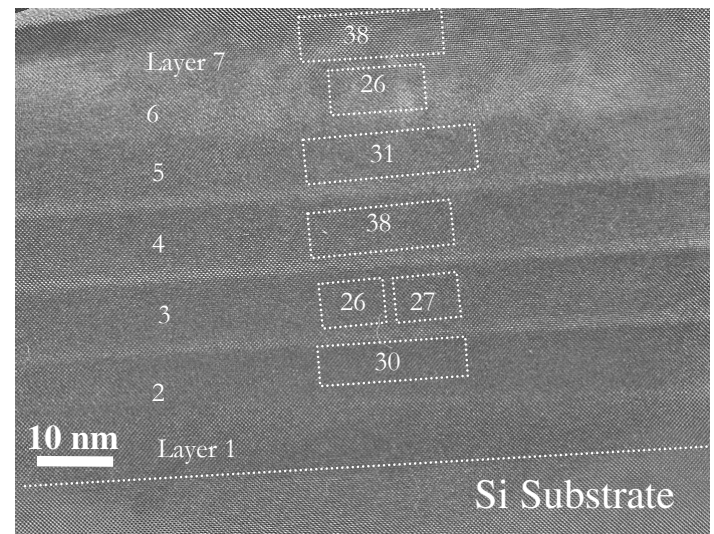

Fig. 9

Average germanium concentration in the centre of stacking islands obtained from the strain map. Averaged areas are shown with white rectangles. 